\title{
Description of Non-Intrusive Sonar Array-Based Technology and its Application to Unique and Difficult Slurry and Paste Flow Measurements
}

\author{
C.V. O’Keefe CiDRA Corporation, United States \\ R.J. Maron CiDRA Corporation, United States \\ P.J. Rothman CiDRA Corporation, United States
}

J. Poplawski CiDRA Corporation, United States

\begin{abstract}
In this presentation, CiDRA's patented technology platform and its applications will be described. CiDRA's non-invasive, passive sonar array-based flow meter technology performs two independent measurements flow rate and fluid characterization. Firstly, the meter provides the volumetric flow rate of the mixture by measuring the speed at which naturally occurring structures such as turbulent eddies or density variations convect with the flow past an axial array of sensors. Secondly, the meter uses similar sonar-based processing techniques and naturally occurring sound in the process slurry to measure entrained air levels and in some cases fluid composition. The result is a unique ability to measure the flow rate and entrained air level of most fluids - clean liquids, high solids content slurries, pastes, and liquids and slurries with entrained air.

Also to be presented is the application of this array-based technology platform in a variety of hydrotransport and minerals beneficiation applications. Examples of these situations include volume flow measurements in tailings lines, thickener discharge, high solids contents pipelines, slurry lines with magnetite and other magnetic ore, slurry lines with abrasive or corrosive materials, high pressure lines, and slurry and nonslurry lines exhibiting scale buildup. The operational advantages and value of these measurements, even in the presence of scale buildup, will be discussed. Recent developments in extending this technology to solve other unique measurement problems such as valve movement confirmation, non-invasive slurry profiling, and sanding detection will be covered.
\end{abstract}

\section{Introduction}

Process control conditions and environments in most minerals processing applications can be challenging. In particular, flow measurement of high solids contents slurries such as pastes or thickened tailings cannot be adequately served by older generation flowmeters such as ultrasonic meters, electromagnetic meters, turbine meters, orifice plate meters, vortex flow meters, Coriolis meters, and venturi meters. Also, in the past, the inability to measure the amount of air entrained within these slurries has degraded the accuracy of nuclear density gauges and flowmeters. Both measurement problems - flow and entrained air - are solved through the use of a new class of instrumentation that utilizes an array of passive sensors and sonar-based processing algorithms to measure flow and fluid composition, including entrained air levels.

\section{Principle of operation}

Sonar array-based meters track and measure the mean velocities of coherent disturbances traveling in the axial direction of a pipe. These disturbances can take many different forms and can propagate at different velocities. Their propagation method and velocities include convection with the flow (slowest velocity), propagation in the fluid or slurry (mid-range velocity), and propagation in the pipe walls (fast velocity). The sonar array-based meters separate the three main propagation modes from each other through a combination of frequency and velocity differences. First let us focus on the disturbances that convect with the flow. 
These disturbances can be density variations, temperature variations, turbulent eddies, or others. Within most industrial processes, these will be turbulent eddies for turbulent flow and density variations for core annular flow. Either type provides an excellent means of measuring the flow rates.

\subsection{Turbulent eddies and flow velocity}

Turbulent flow is composed of eddies, also known as vortices or turbulent eddies, which meander and swirl in a random fashion within the pipe but with an overall mean velocity equal to the flow; that is they convect with the flow. An illustration of these turbulent eddies is shown in Figure 1. These eddies are being continuously created. Once created they break down into smaller and smaller vortices, until they become small enough to be dissipated as heat through viscous effects of the fluid. For several pipe diameters downstream of their natural creation, these vortices remain coherent retaining their structure and size before breaking down into smaller vortices. The vortices in a pipe have a broad range of sizes, which are bracketed by the diameter of the pipe on the largest vortices and by viscous forces on the smallest vortices. On the average, these vortices are distributed throughout the cross section of the pipe and therefore across the flow profile. The flow profile itself is a time-averaged axial velocity of the flow that is a function of the radial position in the pipe with zero flow at the pipe wall and the maximum flow at the center as seen in Figure 1. In turbulent flow, the axial velocity increases rapidly when moving in the radial direction away from the wall, and quickly enters a region with a slowly varying time-averaged axial velocity profile. Thus if one tracks the average axial velocities of the entire collection of vortices, one can obtain a measurement that is close to the average velocity of the fluid flow (Gysling and Mueller, 2004).

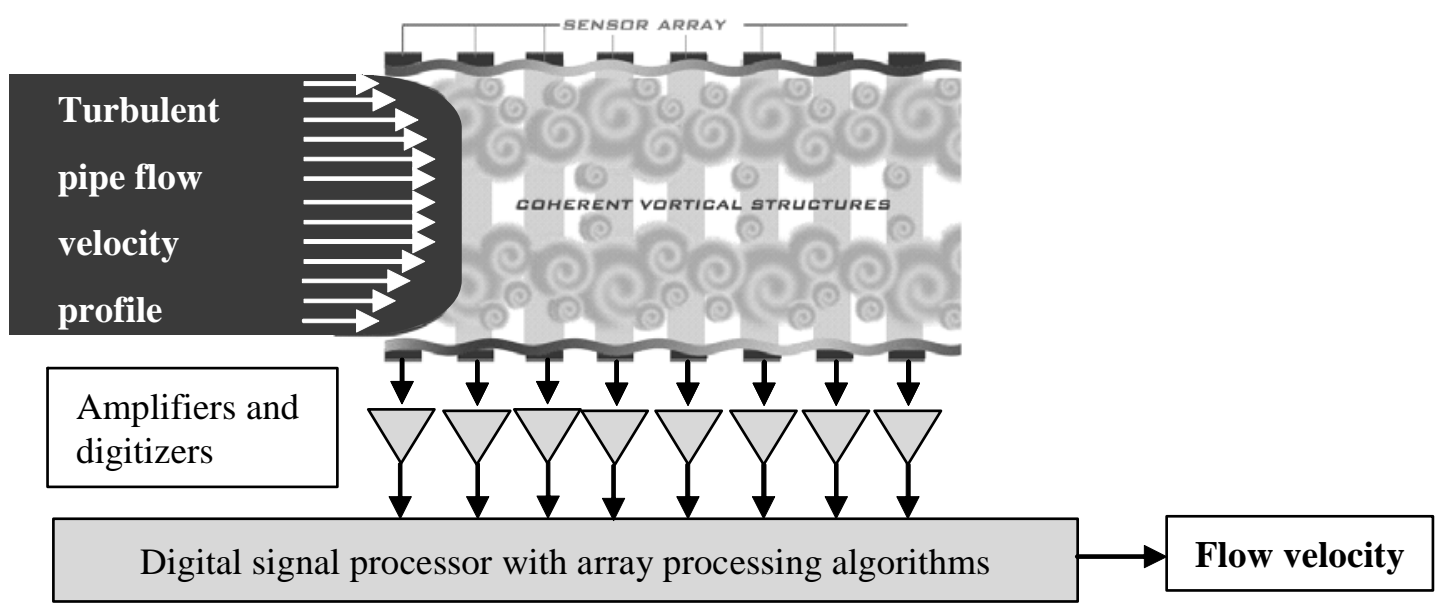

Figure 1 Diagram of pipe with turbulent flow showing fully developed flow profile and turbulent eddies

\subsection{Density variations in slurry/paste and flow velocity}

High volumetric solids content slurries can have viscosities large enough to quickly dissipate any turbulent eddies that may be formed. Fortunately, many of these slurries are shear thinning, and the viscosity decreases as the fluid shears. This results in a core of coarse particles with zero or low settling velocity due to the low shear rate in the center of the pipeline resulting in a high viscosity in this region. Between this core and the pipe wall, there is a high shear rate region, resulting in a low apparent viscosity, thus forming a core-annular flow profile. This shear thinning behavior is advantageous for high solids content slurries transport providing for a lower pressure gradient along the pipeline with its reduced pumping and energy requirements, while presenting a sufficiently high yield stress at the disposal location for proper disposal slopes and stabilities. The core-annular flow profile has non-uniform density variations and ripples at the core to annular interface. These ripples or waves remain coherent for a distance sufficient for tracking through the array of passive sensors (Gysling and Mueller, 2004). 


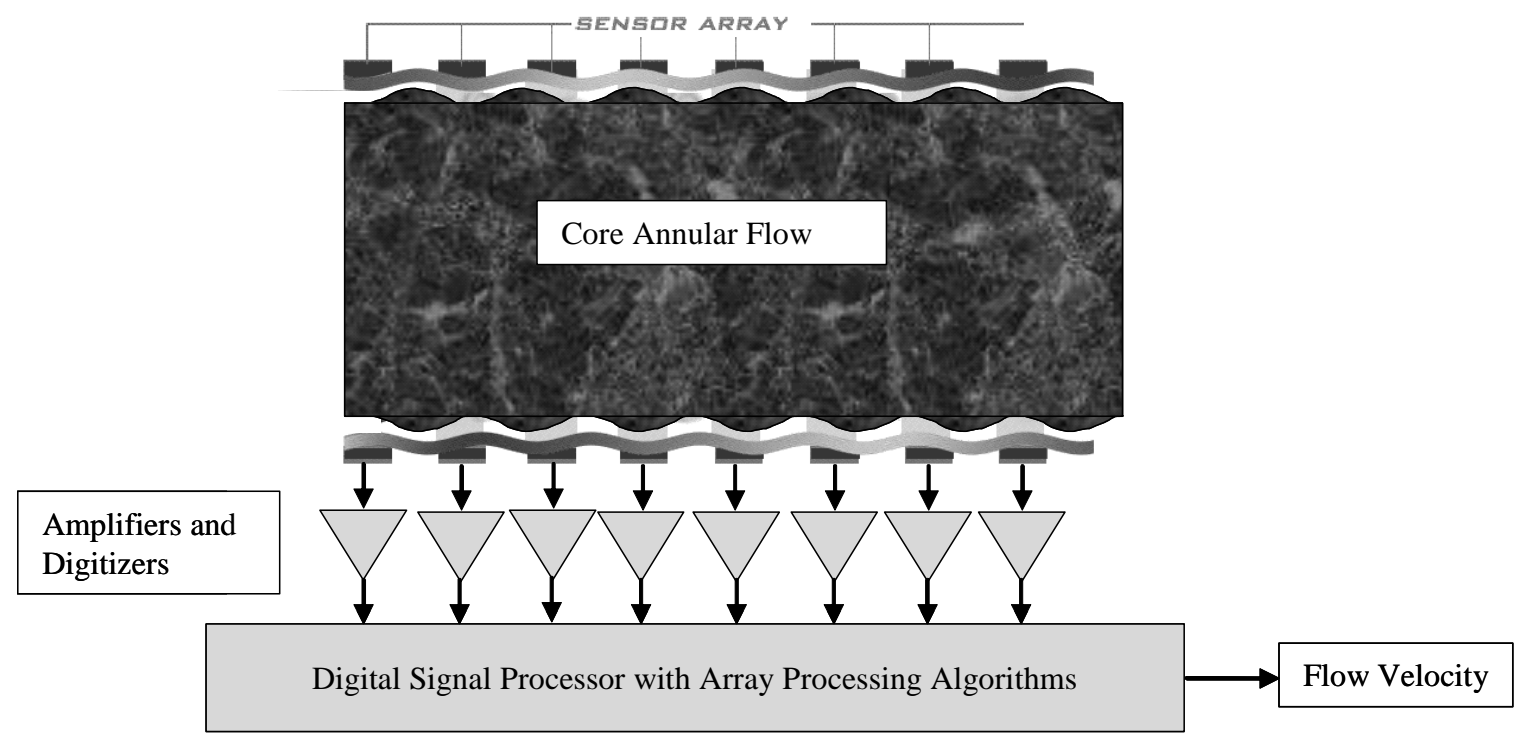

Figure 2 Diagram of pipe showing core annular flow with density variations and waves at core to annular interface

\subsection{Array measurement of flow velocity}

Through the combination of an array of passive sensors and the sonar array processing algorithms, the average axial velocities of a collection of vortices or density variations is obtained. The sequence of events that occur to make this measurement possible is as follows:

- The passage of the turbulent eddies or density variations creates a small pressure change on the inside of the pipe wall.

- This small pressure change results in a dynamic strain of the pipe wall itself (Figures 1 and 2 exaggerate).

- The mechanical dynamic strain signal is converted to an electrical signal through a passive sensor wrapped partially or fully around the pipe - no coupling gels or liquids are required.

- This electrical signal is detected by each element of the array of sensors. These sensors are spaced a precisely set distance from each other along the axial direction of the pipe.

- The resulting electrical signal from each sensor element is interpreted as a characteristic signature of the frequency and phase components of the acoustic waves under the sensor.

- An array processing algorithm combines the phase and frequency information of the characteristic signature from the group of sensor array elements to calculate the velocity of the characteristic signature as it propagates under the array of sensors.

The challenges of performing this measurement in a practical manner are many. These include the challenges of operating in an environment with large pumps, flow generated acoustics, and vibrations, all of which can cause large dynamic straining of the pipe. The impact of these effects is that the dynamic strain due to the passive turbulent eddies or density variations is usually much smaller than the dynamic strain arising from pipe vibrations and acoustic waves propagating in the fluid. The strength in the array processing algorithm is its ability to isolate and measure the velocities of these different components, including the weak signal from the convecting turbulent eddies or density variations, and the strong signals from the acoustic waves and vibrations. The velocity of the acoustic waves is used to calculate the fluid composition or the amount of entrained air (gas void fraction).

The technology lends itself to the generation of a measurement robustness indicator, otherwise known as a quality factor. Most other flowmeters do not provide an indication of the quality of the measurement. 
Conversely, in the sonar processing algorithm such a quality factor can be generated by comparing the strength of the signal from the flow against background energy levels. A quality factor ranging from 0 to 1.0 is generated, with any flow measurement providing a quality factor above 0.1 to 0.2 (depending on the application) having the confidence as being a good measurement.

Currently this technology can report the volume flow rate on liquids and slurries with flow velocities extending from $0.9 \mathrm{~m} / \mathrm{s}(3 \mathrm{f} / \mathrm{s})$ to $9 \mathrm{~m} / \mathrm{s}(30 \mathrm{f} / \mathrm{s})$. The technology lends itself to measurement on practically any pipe size, as long as the flow is turbulent, and in the case of some non-Newtonian fluids, even without turbulence. The pipe must be full to give an accurate volumetric flow rate but it can have entrained air in the form of well mixed bubbles. This technology works on practically any type of fluid within virtually any type of pipe including lined pipes and pipes with scale buildup.

\subsection{Calibration of flow rate and its maintenance}

The volume flow measurement provided by tracking the turbulent eddies does require some adjustment or calibration. In practice the calibration adjusts the reported output by only a few percent, depending on the Reynolds Number. As an example of the correction factor that is applied -seen in the left side of Figure 3 the degree of correction that is required for a DN $250 \mathrm{~mm}(10$-inch) Schedule 10 pipe with water. In this example, after applying the correction factor, the offset is brought to within $+/-0.4 \%$ as seen in the right side of Figure 3.
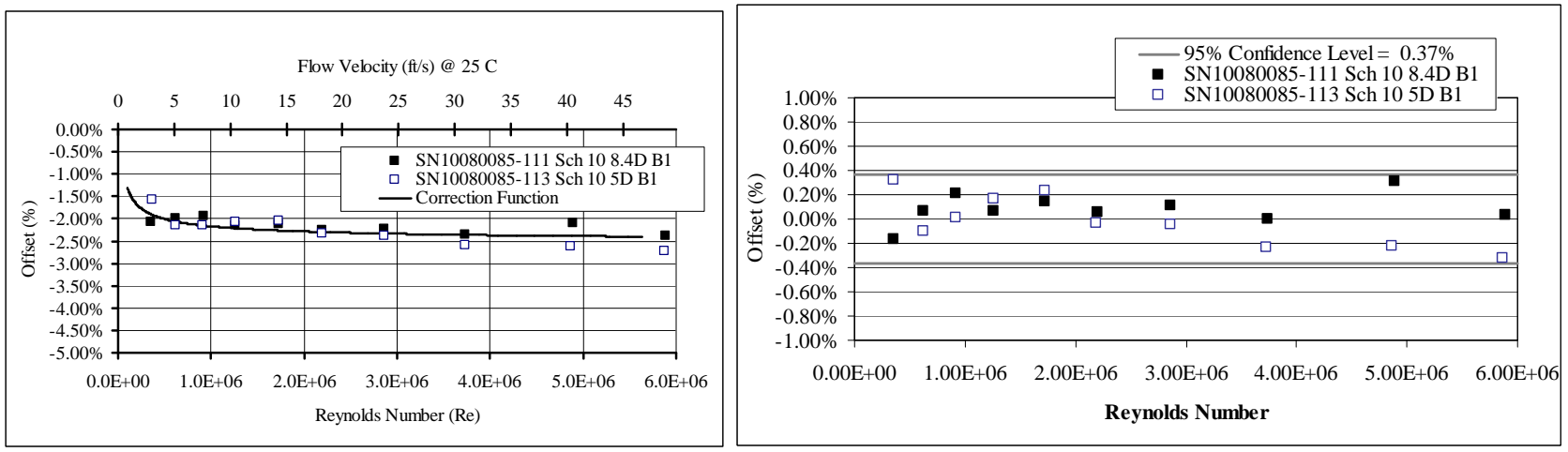

Figure 3 (Left) Flow velocity and offset from reference prior to calibration and (Right) offset after calibration

Since the flow measurement - and hence calibration - is not dependent on the absolute values of any analog signals, it will not drift with time or temperature. Maintenance of the calibration from meter to meter and from temperature effects and aging is dependent on maintaining the spacing between the sensor elements and maintaining the stability of the clock used in the digitizer. The spacing between the sensors is set in the factory where they are bonded to a stainless steel sheet and cannot be adjusted by the customer. The clock stability is better than $0.01 \%$ and thus is 50 times better than the technology's typical accuracy of $+/-1 \%$ in the field; and $+/-0.5 \%$ under reference conditions or after in-field supplemental calibration. As a result the impact of clock stability can be neglected.

\subsection{Maintenance of calibration between water and slurries}

The sonar-based flowmeter is well suited to measuring aggressive slurry flows with variable solids content. Figure 4 shows a volumetric flow measurement during a transition from water to iron ore concentrate slurry (s.g. $=2$ ), while the positive displacement pump maintained a constant speed. During this transition period, the standard deviation of the volumetric flowrate was less than $1 \%$ of the average flowrate, thus showing very good insensitivity to large density changes. Other tank fill or tank drawdown tests have verified the use of the same calibration coefficients for fluids ranging from water to thick slurries including a sand paste with a mass weight of $67 \%$. 


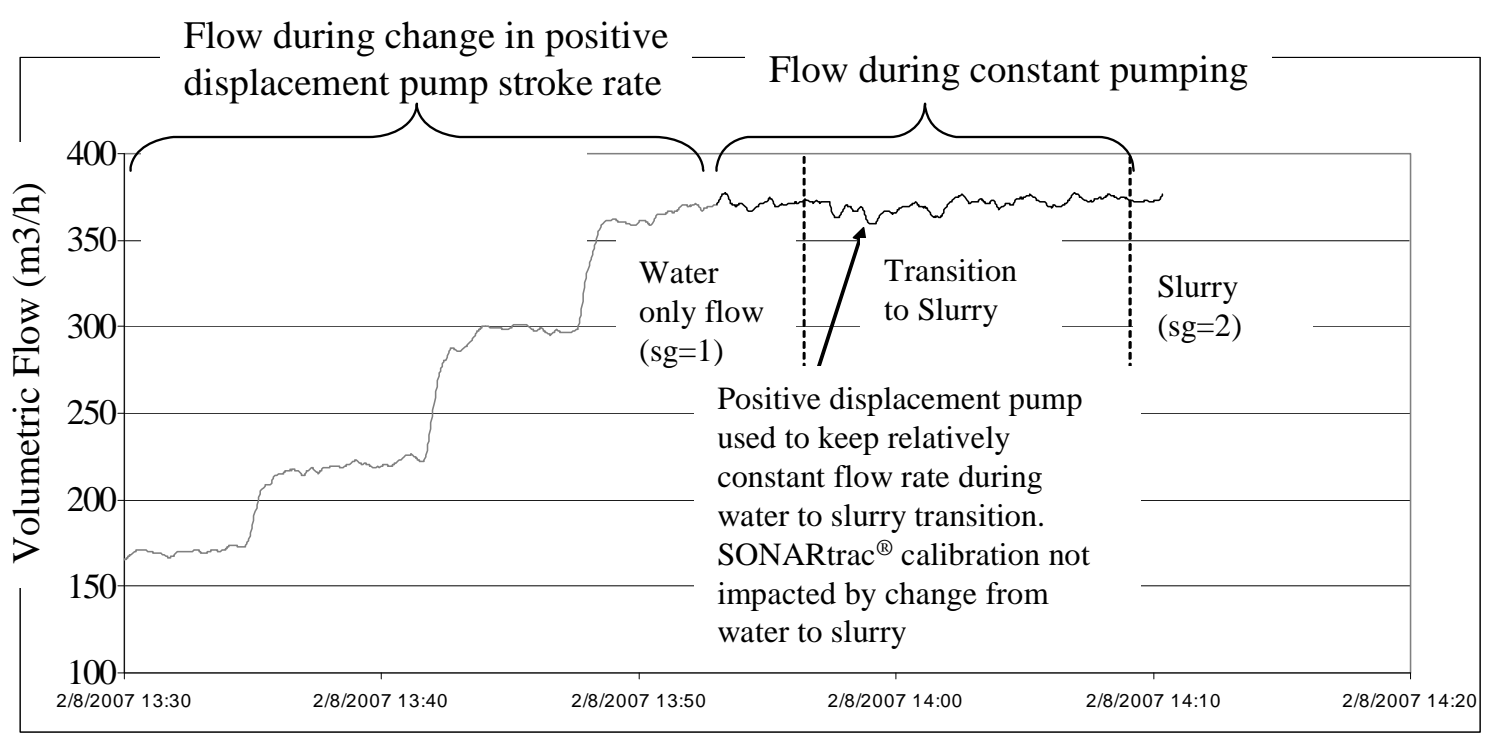

Figure 4 Measurement of both water and iron ore concentrate slurry with same meter and with same calibration coefficients show no changed in measured flow. Use of a positive displacement pump keeps flow rate the same

\subsection{Fluid composition measurement}

In most mineral processing processes including paste or thickened tailings applications, there is an abundance of acoustic waves propagating within the pipes. These acoustic waves are generated naturally from a variety of sources, including pumps, the flow-through devices, and the flow-through pipe geometry changes. Even bubbles within the fluid will generate acoustic waves through their natural oscillations. These acoustic waves are low frequency (in the audible range), and travel in the pipe's axial direction, with wavelengths much longer than the entrained gas bubbles. An illustration of these acoustic waves in a pipe is shown in Figure 5 and, as can been seen in the figure, they can propagate in either direction down the pipe or in both directions (Gysling et al., 2005).

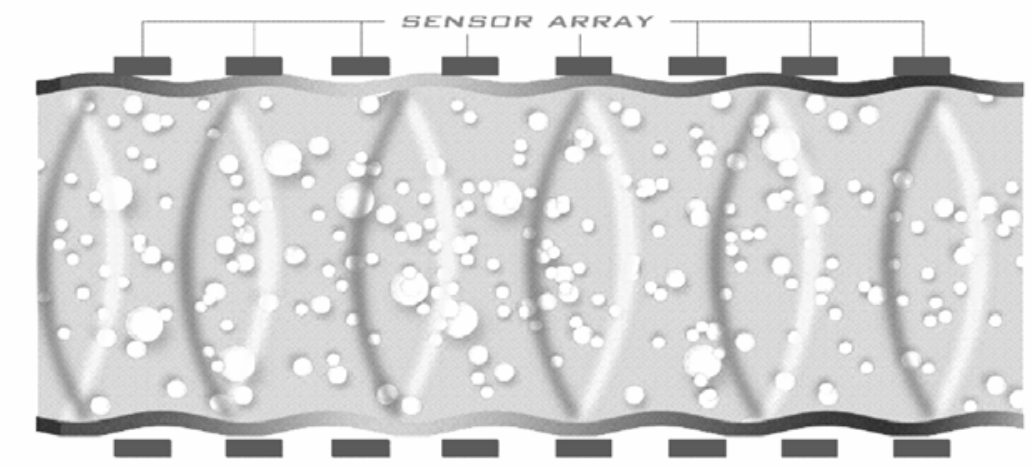

Figure 5 Illustration of naturally occurring acoustic waves propagating in pipe under the sonar array sensors

Through the same array of passive sensors used to measure the flow velocity and similar sonar array processing algorithms, the average axial velocities of a collection of acoustic waves is obtained. Since acoustic waves are traveling pressure waves, they introduce localized pressure changes on the inside of the pipe walls during their cycling from compression to rarefaction and back. These pressure changes strain the pipe walls and are tracked in a similar manner as for the turbulent eddies or density variations.

This fluid can be multiphase, or multicomponent single phase. In a single phase fluid, the acoustic velocity is a function of the ratio and acoustic properties of the two fluids, thus this measurement can be used to 
determine mixture ratios through application of the simple mixing rule (volume average of velocity). The resulting acoustic velocity $c_{\mathrm{M}}$ can be given by:

$c_{M}=\phi_{1} c_{1}+\phi_{2} C_{2} \quad$ (Wang and Nur, 1991) where $\phi_{1,2}$ are the phase volume fractions and

$c_{1,2}$ are the acoustic velocities of the phases

Using $\phi_{2}=1-\phi_{1}$ this can be rearranged to give: $\phi_{1}=\frac{C_{M}-c_{2}}{C_{1}-c_{2}}$

In multiphase fluids that consist of a gas mixed with a liquid or slurry, the acoustic velocity can be used to determine the amount of entrained gas (gas void fraction) when the gas is in the form of bubbles that are well mixed within the liquid or slurry (Wood, 1930).

Since the wavelengths of the acoustic waves are much larger than the bubble size, a complex interaction takes place that sets the acoustic velocity to be a strong function of the gas void fraction. The speed of sound is proportional to the square root of the ratio of the compressibility and the density, both of which are heavily influenced by air content. An example of the resulting relationship is shown in Figure 6.

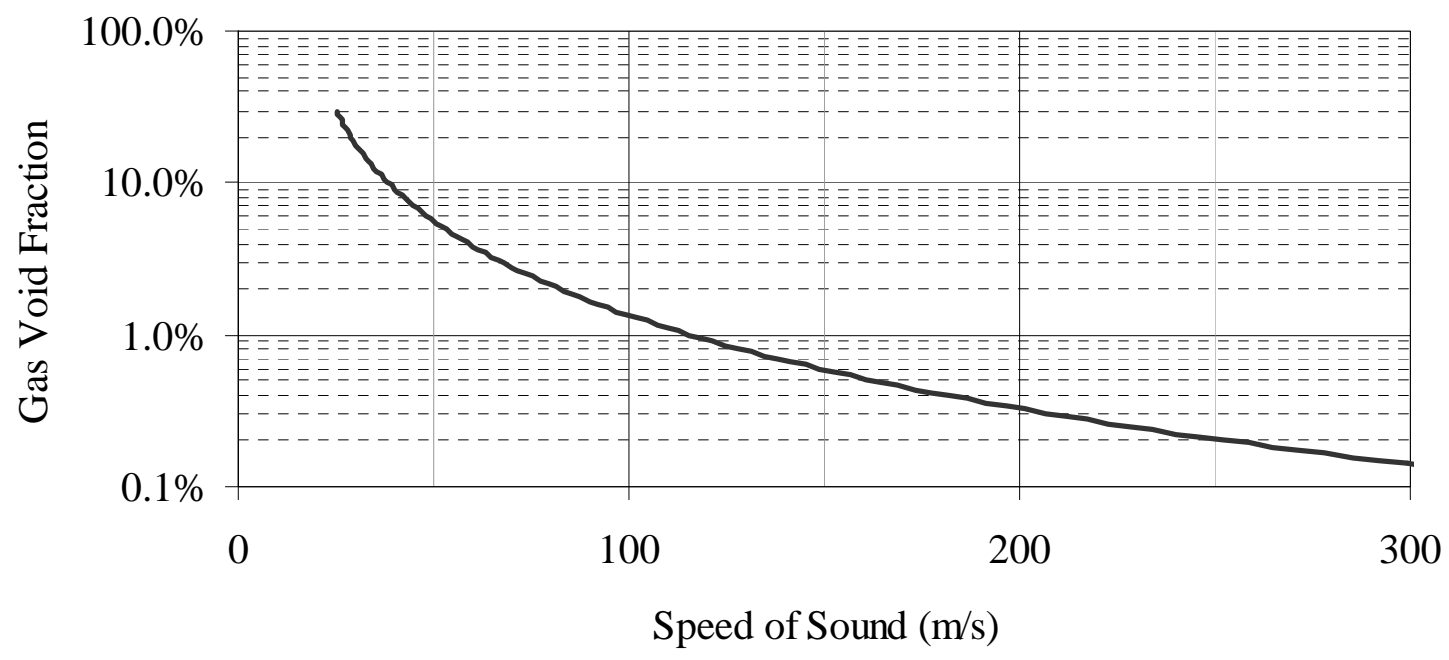

Figure 6 Example of relationship between gas void fraction (entrained air content) and speed of sound

The gas void fraction measurement is used in a variety of different fields and applications. Within mineral processing, it is used for nuclear density gauge correction, flowmeter correction to provide true volume flow, diagnosis of pumping issues, detection of flashing, and air injection applications. It is being successfully used for entrained air applications ranging from $0.01 \%$ to $20 \%$ gas void fractions with an accuracy of $5 \%$ of the reading (Gysling et al., 2005).

\subsection{Hardware implementation of measurement principle and installation process}

The flow and fluid composition measurement principle can be implemented through a variety of hardware configurations. To take advantage of the non-invasive capability of this technique, an externally mounted, wrap-around instrument was developed. To understand this hardware implementation, it is best to see the installation process as seen in Figure 7. There are no coupling gels required and the sensors do not require alignment. The installation is easy, usually requiring one person and typically takes two hours. 

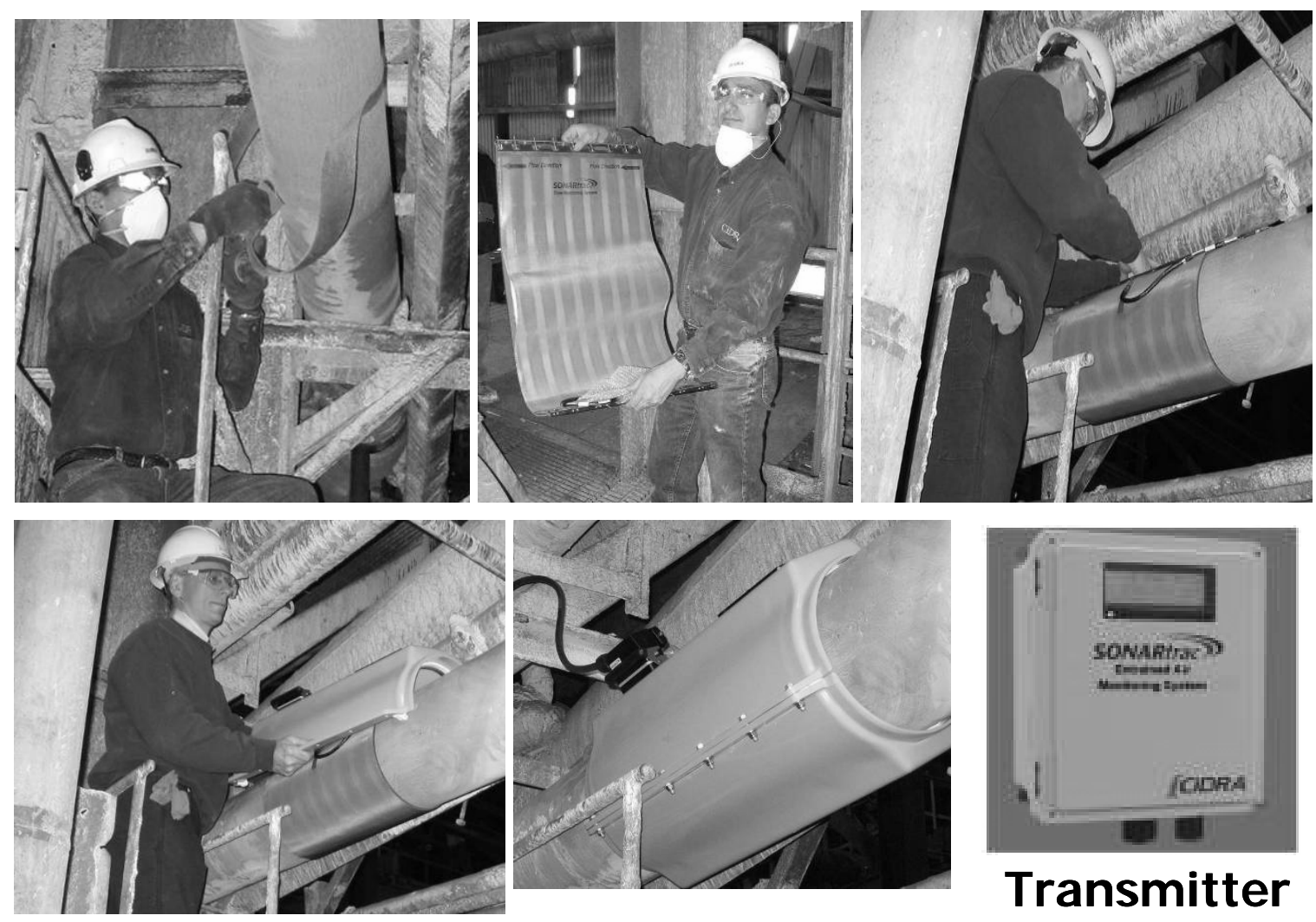

Figure 7

Installation procedure from (top-left) pipe prepation through cleaning and light sanding of pipe to (top-middle and top-right) mounting of the flexible, lightweight sensor band to (bottom-left) installation of ths sensor cover and to (bottom-middle and bottom-right) connection of sensor cover to transmitter via water tight cable

\section{Measurement solutions}

This technology can be used in most applications in which older generation flowmeters such as electromagnetic, differential pressure and ultrasonics flowmeters are used. It also excels in many applications in which these other flowmeters do not function well or at all, have limited lifetimes, or pose a safety risk. These include highly abrasive/corrosive fluids, high pressure lines, slurries with entrained air, pipes with scale buildup, slurries with magnetic ore, and others.

\subsection{Accurate non-invasive flow measurements for high pressure applications and leak detection}

Due to its non-invasive nature and easy installation, the sonar array-based flowmeter is ideally suited for abrasive and/or high pressure applications. As an example, there was a need to have a reliable flowmeter to measure flow at the beginning and end of a $>50 \mathrm{~km}$ pipeline. The requirement was to accurately measure flow in order to detect any leaks, as well as monitor the load out rate. The challenge for the plant was to do so without breaking into the pipe due to the high pressures ( $>70$ bar) seen on the second flowmeter site. A picture of the high pressure installation (Figure 8) shows how the external nature of the flowmeter makes for a quick and safe installation, as well as safe operation.

The resulting flow measurements seen in Figure 9 clearly show the two flowmeter signals (dark lines) lying on top of each other. The only way to see the small differences between the two readings is by looking at the ratio of the two outputs (light line). Except where transitions cause a difference in flow between the top and bottom meters due to the transit time of the flow change in the pipeline, the averaged ratio is within approximately $+/-1 \%$, which is within the specifications of the meters and the requirements of the plant. 

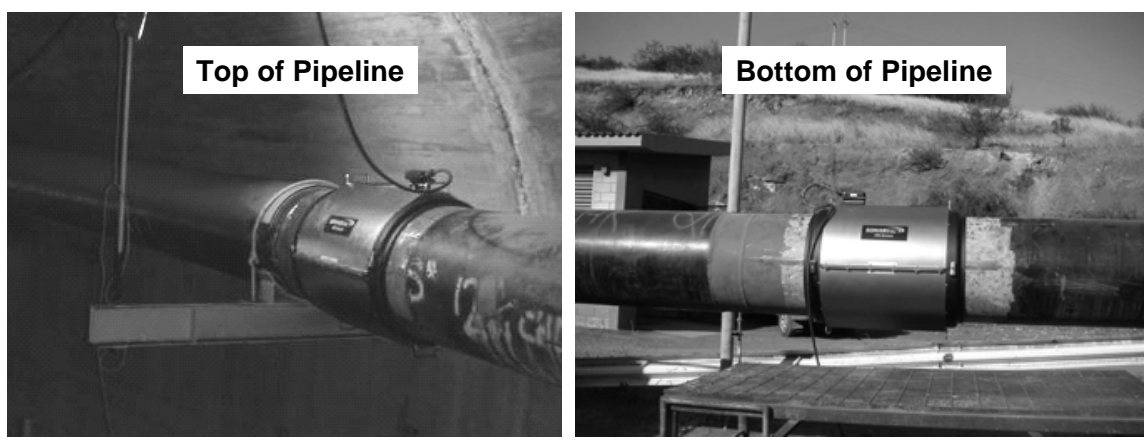

Figure 8 Safety in high pressure lines ( $>70$ bar), using the same meter for both high pressure and low pressure applications

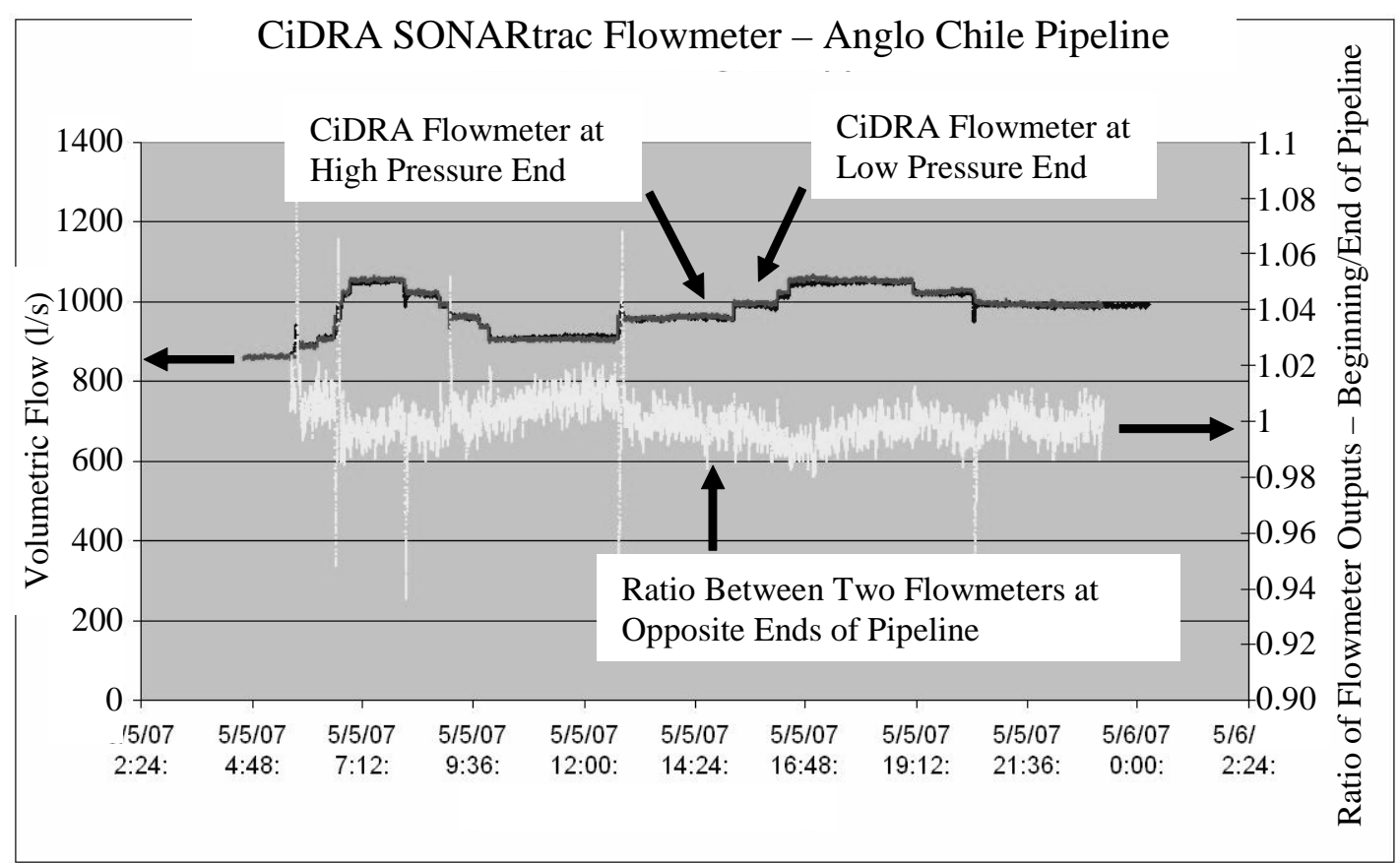

Figure 9 Results of leak detection using sonar array-based flowmeters in which overlapping dark lines are flowmeter outputs and the light line is the ratio between the two outputs

\subsection{Accurate flow measurement without drift}

There are many cases where the measurements provided by flowmeters cannot be verified through an accurate gold standard test such as a tank fill or draw down calibration. Most flowmeters will drift with time and/or temperature resulting in a change in the signal that is not noticed or cannot be verified. As an example, magmeters rely on the stability of analog electronics that can drift with time and temperature, the absence of magnetic particles in the ore, and/or clean electrodes to accurately report flow. When any of these conditions are not met, which happens frequently, the operator is not even aware that an error has taken place unless the magmeter is compared to another meter, or is recalibrated via a gold standard test.

As an example, data is shown in Figure 10 from two magmeters placed in series in close proximity to each other at a gold and copper mill. In that figure the two dark lines are the magmeter outputs, while the light line between the two dark lines is the sonar array-based flowmeter. The sonar array-based flowmeter was configured using the universal calibration coefficients used for this meter. Here the two magmeters differ on the average by over $12 \%$. The data from a sonar array-based flowmeter is seen to provide a flow reading that is approximately an average of the two magmeters, but with the confidence that it will not drift with time. Maintenance of the relative accuracy can be verified by moving the sonar array-based flow meter, without stopping the process, to a location where a tank test can be performed. 


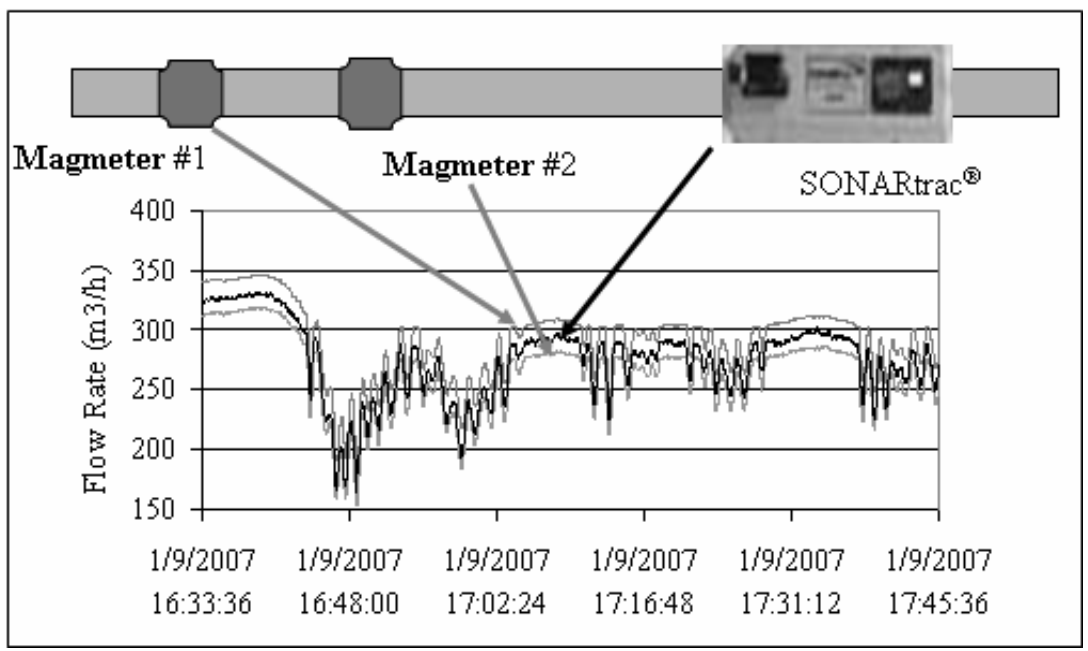

Figure 10 Two magmeters in series with sonar array-based flowmeter showing offsets $>12 \%$ between two magmeters and accurate measurement provided by sonar array flowmeter

\subsection{Measuring flow in the presence of magnetic ore such as magnetite, arsenopyrite or pyrrhotite}

Magnetic ore in a slurry line, whether intentional in an iron ore mill or whether unintentional in mills concentrating other metals, poses a potential problem for magmeter flow measurements. Quite a few locations mining copper, gold or other non-ferrous metals have magnetic ore in or near their ore body. The magnetic ore, even in small quantities, changes the magnetic field within the magmeter and can cause the magmeter to register a higher flow rate than the actual flow rate, or introduce a high quantity of noise in the flow rate output. Magmeter manufacturers have attempted to circumvent the impact of magnetic ore with a third coil, with magnetic field measurements, and with manual offset adjustments based on laboratory samples of the typical slurry. These methods have resulted in mixed results in which many times, the calibration or offset changes depending on the quantity of magnetite present.

A better solution is to use a flowmeter technology that is not impacted by the presence of magnetite. Since the passive array technology used in the sonar based flow monitoring system does not rely on the use of any magnetic fields, it is totally impervious to the effects of magnetite. An example of this is illustrated in Figure 11 in which a sonar-based flowmeter is compared to a magmeter. In the figure, one can see that as the density of the magnetic ore increases, the magmeter erroneously reports a higher flow rate, whereas the sonar based flowmeter correctly continues reporting no change in the flow rate.

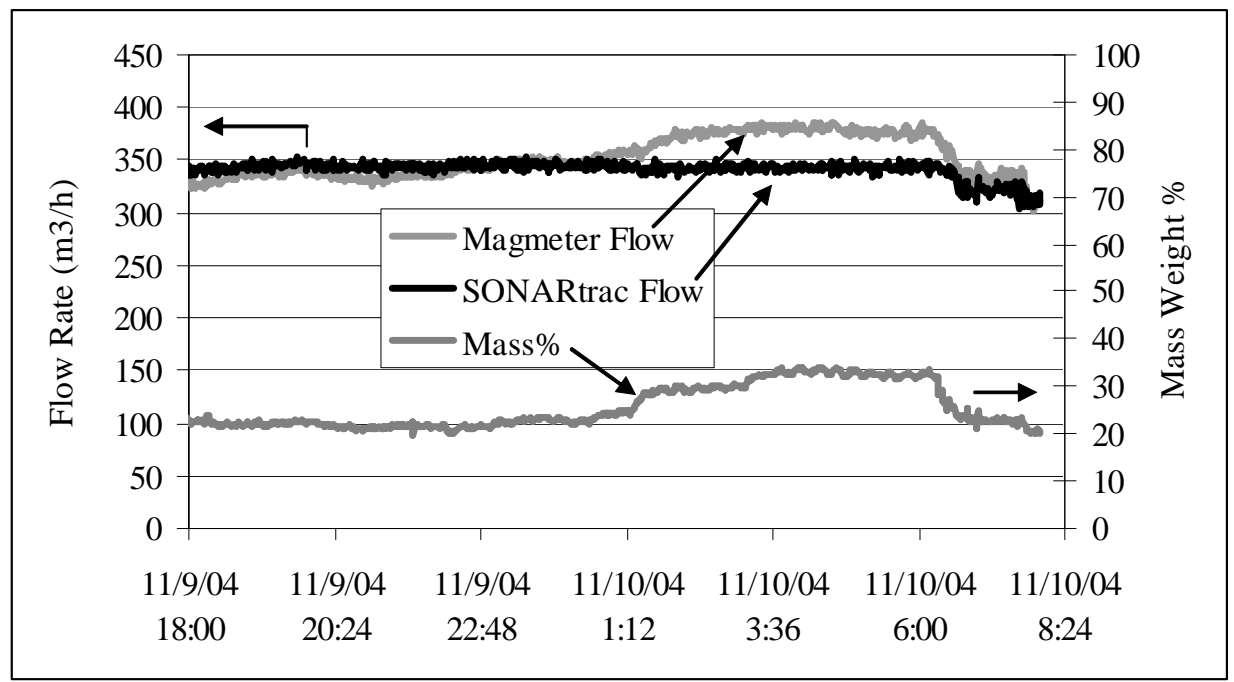

Figure 11 Magmeters erroneously respond to magnetite while array based flowmeter accurately reports flow 


\subsection{Flow measurement of slurry containing entrained air}

Air can enter a slurry pipeline intentionally through gas injectors used to reduce frictional losses, or unintentionally through leaks, filter based thickening processes, or cement injection. In terms of volumetric flow measurement, most flowmeters are adversely affected by air entrained within a liquid or slurry. As a minimum, they cannot provide the true liquid or slurry flow, while in many cases the entrained air will cause a large increase in flow meter noise or a total loss of flow readings. The ability of the array based technology to measure flow in the presence of high levels of entrained air as well as the entrained air level itself, lead to better control of the process.

Northgate Minerals uses CiDRA Corporation's sonar array-based (SONARtrac ${ }^{\circledR}$ ) technology to measure bulk concentrate flows and the feed to the flotation columns at Kemess mine in British Columbia, Canada. The non-intrusive technology measures these abrasive slurries accurately with no process downtime because of maintenance issues with the flow meter. Kemess mine also uses the Gas Void Fraction capability of the sonar array-based meter as a tool to monitor increases of air in these lines. Increased air will cause pump inefficiencies and could lead to pump damage due to cavitations. Knowing the volume of entrained air provides a true volumetric flow rate and provides operators with another tool for process control. In Figure 12 the step increase of entrained air from approximately 6\% to 8\% coincides with the decrease in flow from 2300 GPM to 1900 GPM. This may indicate that the increased entrained air is impacting the operation of the pump and alert the operator to a condition that needs attention

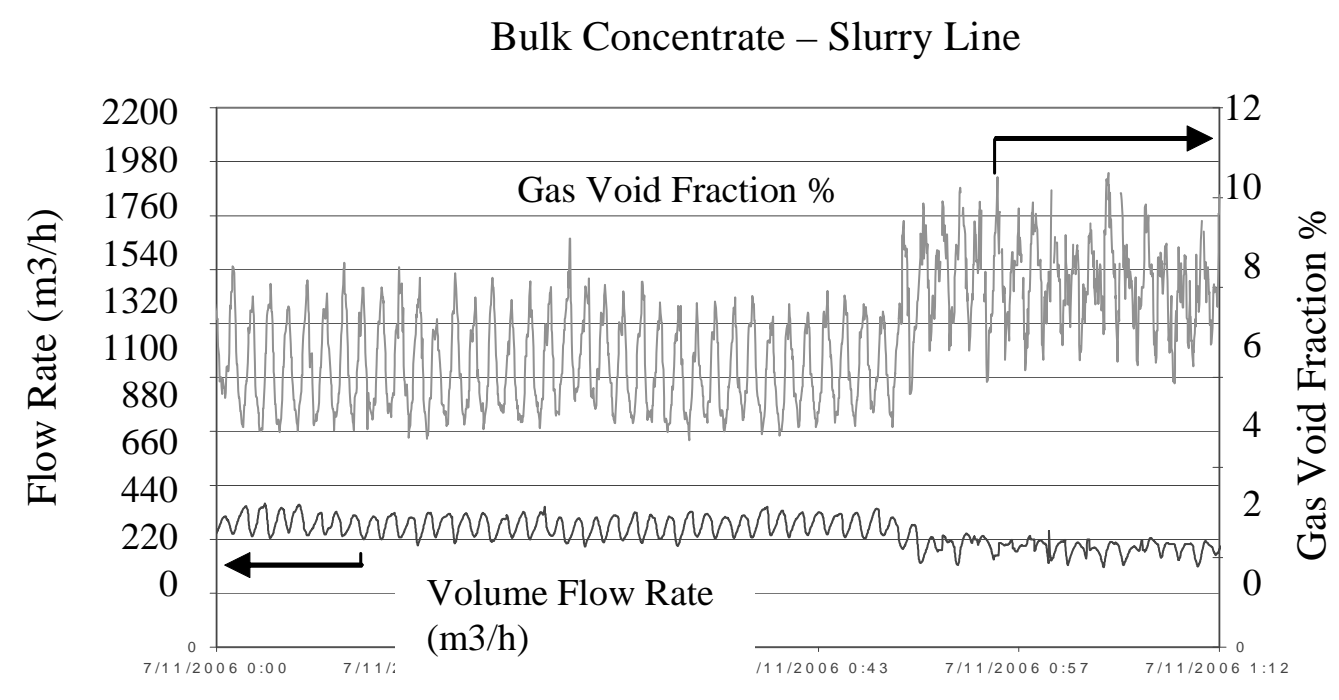

Figure 12 Flow and entrained air (gas void fraction\%) measurements show impact of entrained air on flowrate and technology's ability to measure both

\subsection{Correction of nuclear density gauges due to entrained air}

The presence of entrained air or gas void content will directly reduce the specific gravity reported by a nuclear density gauge. In order to obtain the correct density measurement of the slurry itself, the gas void fraction must be measured and used as a correction factor. To validate this approach, we ran a test in which varying levels of air were introduced into a flow loop containing a nuclear density gauge. As expected, when the air injection rate, shown as standard cubic meters per hour in Figure 13, was increased the nuclear density gauge output seen in the light solid line decreased. The sonar array-based flowmeter on the same line accurately measured the resulting air content as seen in the dashed line. Using this measurement, we applied a simple linear correction of the nuclear density gauge output to reduce the error from $5 \%$ down to $+/-0.25 \%$. 


\section{Gas Void Fractions up to 5\%}

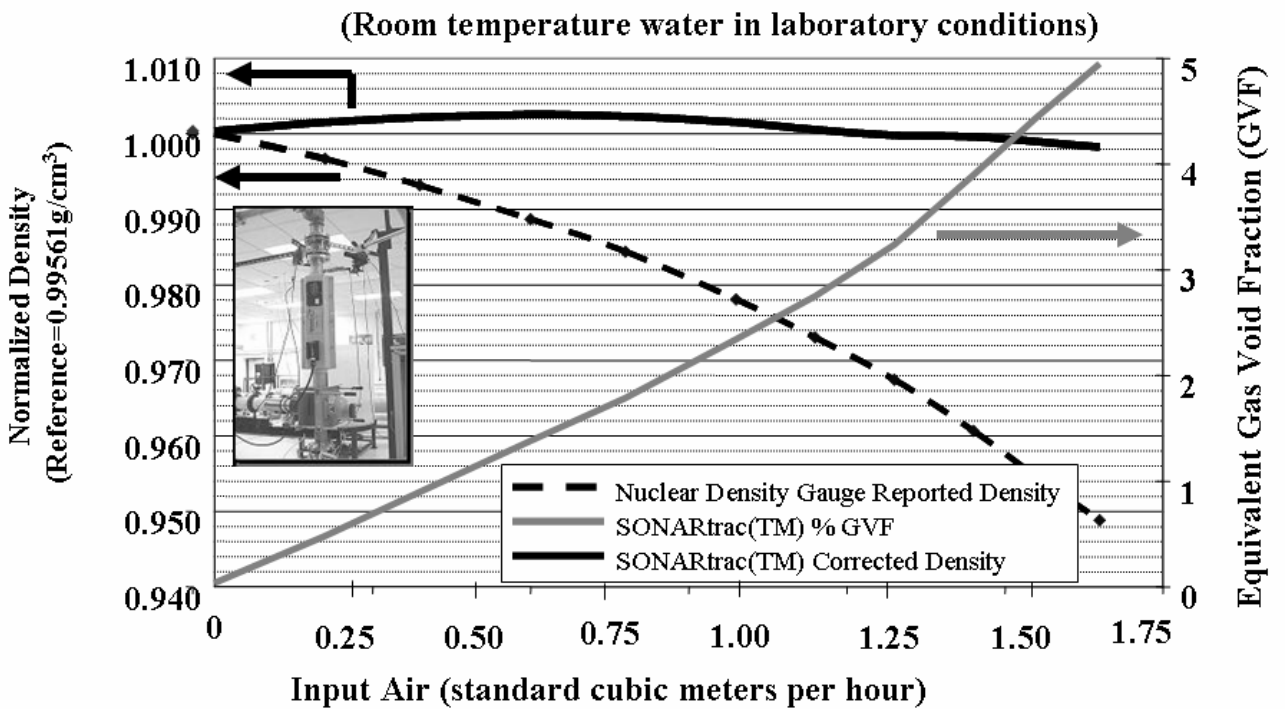

Figure 13 Nuclear density gauge correction using sonar-array based meter

\subsection{Thickened tailings flow}

As previously described, the sonar-based flowmeter is also capable of measuring non-Newtonian, shearthinning slurries. In the mining industry, examples are high-solids content slurries commonly known as thickened tailings and paste. Figure 14 shows an example of a sonar-based flowmeter measuring a thickened tailings flow at low velocities below $1 \mathrm{~m} / \mathrm{s}$. As the flow transitioned from water at over $2 \mathrm{~m} / \mathrm{s}$ to slurry, the velocity immediately decreased to $1.2 \mathrm{~m} / \mathrm{s}$ and then continued a slow additional decrease until the line completely plugged at $0.5 \mathrm{~m} / \mathrm{s}$. Subsequently, the line was unplugged and cleaned with water flow as shown. This sequence shows that with such slurries, the high density produces a sufficiently high amplitude selfgenerated coherent pressure field that can be tracked by the sonar-based flowmeter. For this test, conducted for Antofagasta Minerals S.A., Chile, by PSI-JRI Ltda. (both of Chile) the accuracy of the sonar flowmeter was verified by tank-fill tests as shown on the graph.

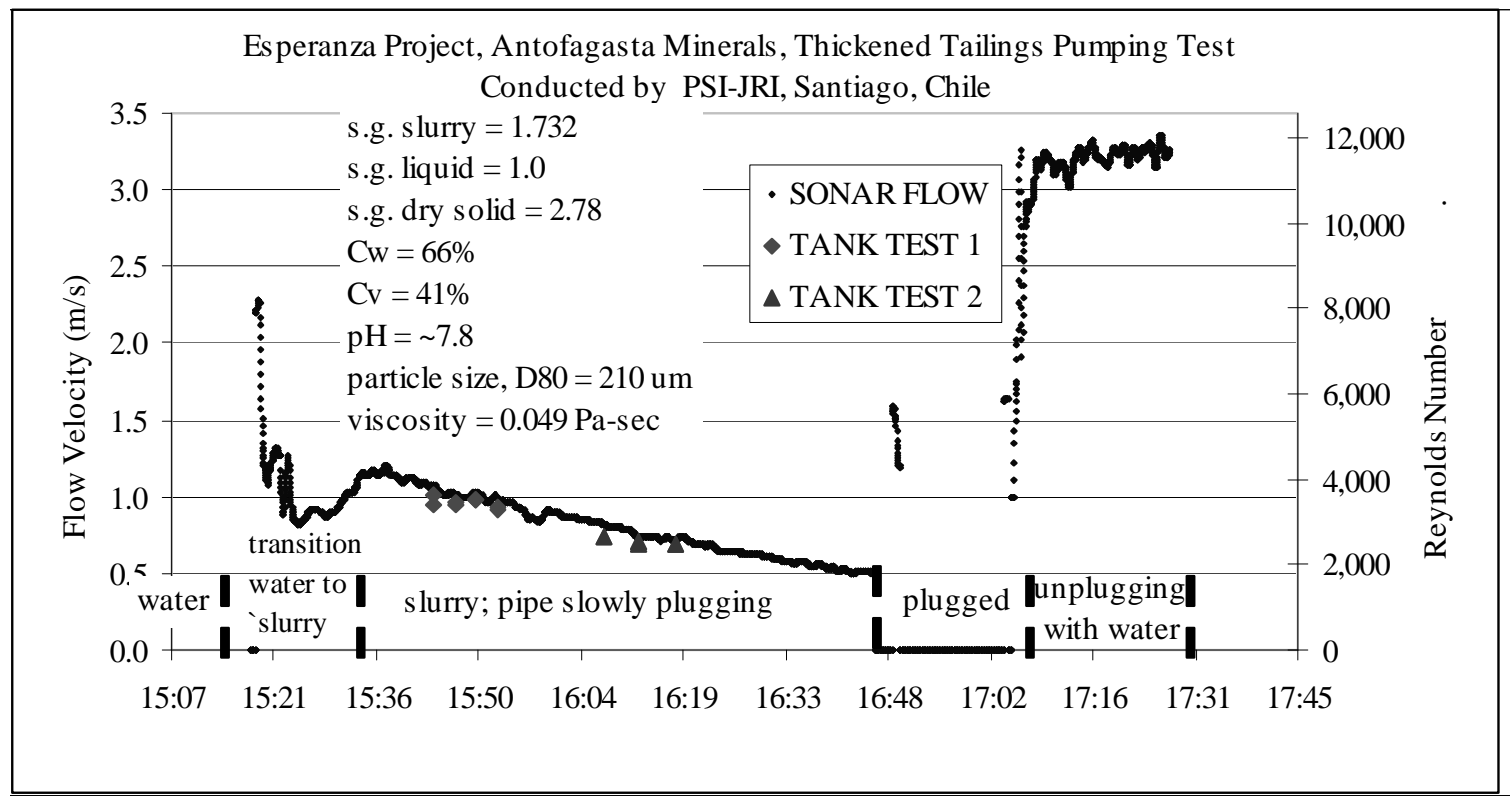

Figure 14 Thickened slurry test for Antofagasta Minerals, Chile, with flow measurements using sonar array-based flowmeter showing accuracy verified through tank fill tests and ability to operate at flow velocities below specifications 


\section{New measurement capabilities}

The array-based technology leads itself to other measurement capabilities. Many of these features can be implemented on the same hardware using only a software change. By using the passive sensors in this hardware implementation as acoustic sensors it is possible to monitor pipelines for ruptures, valves for movement, and pumps for cavitation, impending failures or other machine diagnostics. Some of these capabilities are still under development whereas some, such as valve movement monitoring in choke stations, have been incorporated in the meter. The speed of sound measurement has been used to not only determine the amount of entrained air, but also to distinguish between slurry flow and water flow in batch operations. Some new measurement capabilities do require specialized hardware. One example is flow profiling and sanding detection. Through a different hardware configuration, the flow profile in a horizontal pipeline can be determined, leading to the detection of a sanding out condition which can lead to pipeline plugging.

\subsection{Acoustic analysis for pipeline and valve monitoring}

During the course of measuring flow, the passive sonar-array based flowmeter developed by CiDRA detects the acoustic levels within the pipe. By monitoring these acoustic levels over selected frequencies, additional information about events occurring in a pipeline can be obtained. As an example, valve movement in a pressure reduction choke station corresponds with changes in the acoustic levels during the movement, as well as before and after the movement as the flow is diverted through a different pipe. The flow shown as the dark line in Figure 15 changes by about $8 \%$ due to a change in the valve position which directs the flow through a different path in the choke station. The acoustic level changes by a factor of three to four (200\% to $300 \%)$ during the valve movement and by a factor of three (200\%) between valve positions. The combination of the flow measurement and acoustic level provides the necessary information to monitor the valve. This concept can be extended to monitoring of bursting disc events, and for pipeline breaks.

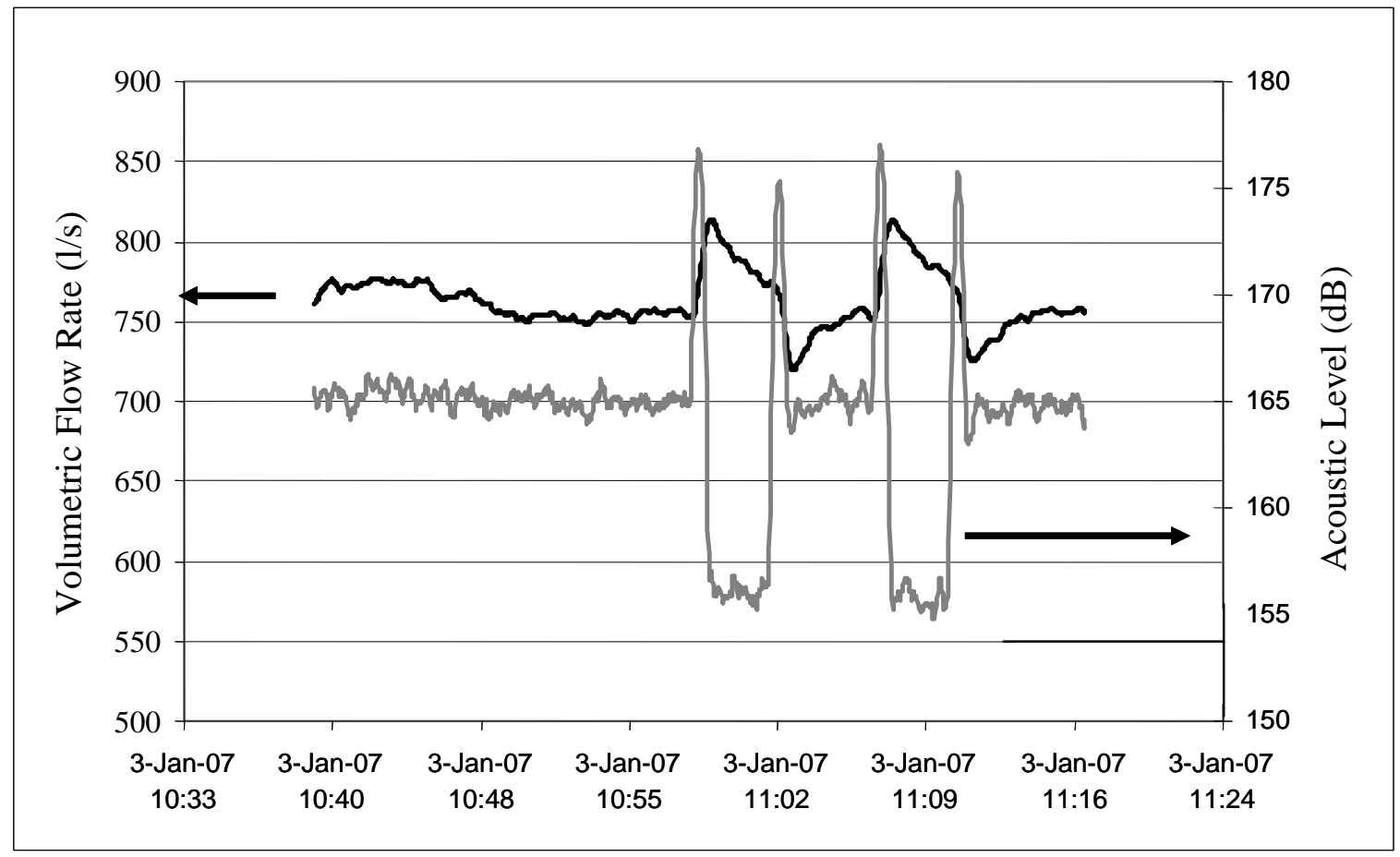

Figure 15 Flow measurement and acoustic level measurement for valve movement monitoring at choke station

\subsection{Batch tracking using real-time speed of sound measurement}

A unique ability of the sonar-based flowmeter is its ability to measure the real-time speed of sound in process piping. Figure 16 shows simultaneous measurements of volumetric flow and speed of sound from two sonar-based flowmeters located $26 \mathrm{~km}$ apart on a hydrotransport slurry pipeline. At the start and end of a 
multi-hour batch run, and sometimes during the batch, water is typically introduced for a short time. The graph shows a clear change in speed of sound between the water and slurry, enabling detection of the water batch at the two meters which have a transit time between them of approximately three hours. The noninvasive nature and insensitivity to pressure of the sonar-based flowmeter allowed installation of one meter at a high-pressure location ( 70 bar), at the discharge of a large positive displacement pump, without stopping the process.

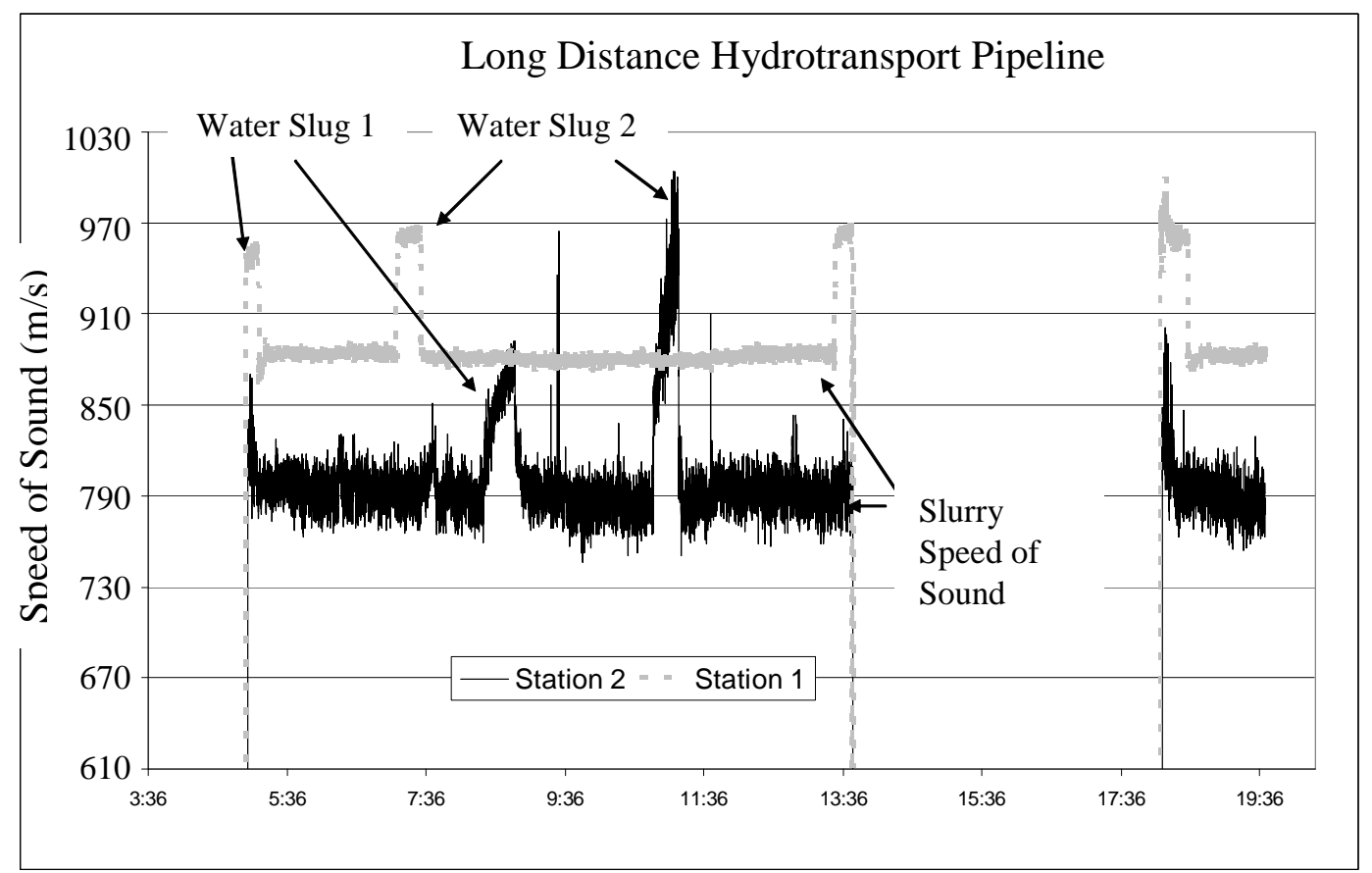

Figure 16 Detection of water slugs separating batches of concentrate in hydrotransport pipeline at two different locations on pipeline

\subsection{Flow profiling and sanding detection}

One key problem that continues for operators of hydrotransport pipelines is the possibility that solids material will settle to the bottom of the pipe and lead to a blockage in the pipe. Operators strive to avoid this "sanding out" condition by keeping flow rates above a certain empirically determined or calculated value. Unfortunately, incomplete models and changes in the slurry properties such as viscosity and particle size distribution (PSD), result in large error bars with either approach.

A better solution is to actively monitor the flow profile in the pipe to see the reduction in flow velocity at the bottom of the pipe as the larger, denser particles settle and approach a very slow velocity. Using a different instrument and a specially engineered sensor, the flow velocity may be determined at locations across the diameter of the pipe. As an example, the flow at the bottom of the pipe may be compared to the flow at the top of the pipe. Results from testing this custom instrument are shown in Figure 17. Here the change in the flow profile due to the stratification of particles is evident in the lower velocities seen near the bottom of the pipe as the "sanding out" condition is approached.

By processing some characteristic features of the flow profile, an alarm condition can be generated. By extending this processing to examine not only the condition where a small level of "sanding out" has occurred but has now reached a higher level in the pipe (>25\% of the pipe height) we can set a higher level in the alarm. This is illustrated in the right side of Figure 17. 

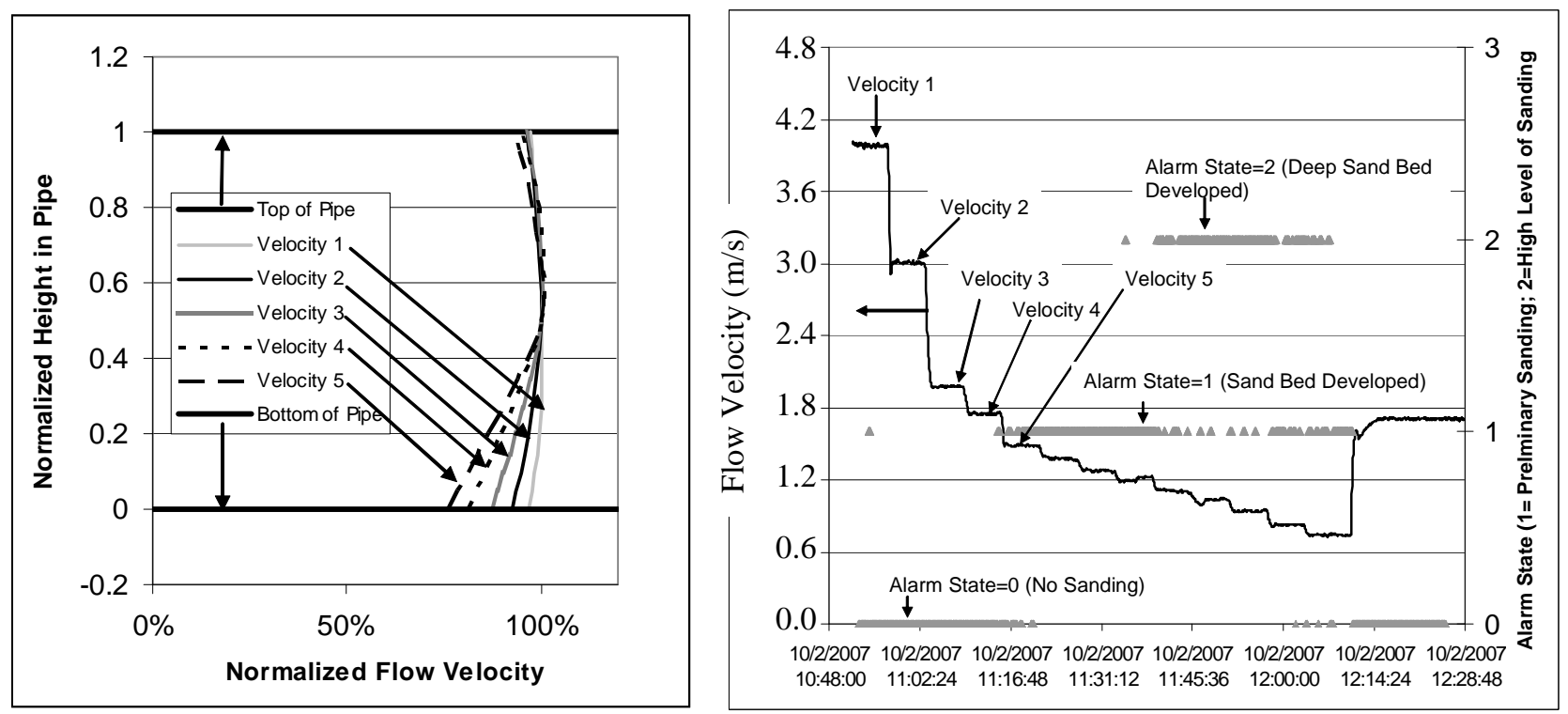

Figure 17 (Left) Normalized velocity profiles at various flow rates showing a drop in velocity at the bottom of the pipe at the lower flow rates. (Right) Flow velocity $\&$ alarm condition for low levels of sanding (Alarm level=1) and high levels of sanding (Alarm level-2)

\section{Summary}

Sonar array-based meters have demonstrated new advantages in flow measurements and new measurement capabilities. This technology has now been fielded in fourteen countries at forty-five mining locations with over 300 meters installed. The advantages of this unique measurement technology have been illustrated in this paper for applications including thickened tailings, high levels of entrained air, high and low pressure pipelines, leak detection, and magnetic ore slurries. Besides performing accurate flow measurements under difficult conditions, this technology has the ability to perform other types of measurements. Some of these measurements including acoustic monitoring of valve movements, distinction between slurry and water in a pipeline, and entrained air were illustrated.

\section{Acknowledgements}

The authors wish to thank our customers for adopting the SONARtrac ${ }^{\circledR}$ flow and process meters, providing data and other support. For this paper, these include, but are not limited to Newmont Gold, Anglo American Chile, Antofagasta Minerals, PSI-JRI Ingenería Ltda., and Kemess Mine. The authors wish to thank Antofagasta Minerals S.A. and PSI-JRI of Santiago, Chile for allowing the inclusion of thickened tailings data. The authors wish to acknowledge the work performed by mineral processing team members at CiDRA including Tim Griffin for analyzing data and performing the nuclear density gauge tests, Mark Fernald and Tim Bailey for performing the velocity profiling tests.

\section{References}

Gysling, D. and Mueller, E. (2004) Application of Sonar-Based, Clamp-on Flow Meter in Oilsand Processing, ISA 2004 Exhibit and Conference.

Gysling, D., Loose, D. and van der Spek, A. (2005) Clamp-on, Sonar-Based Voumetric Flow Rate and Gas Volume Fraction Measurement for Industrial Applications, Flomeko 2005 Conference.

Wang, Z. and Nur, A. (1991) Ultrasonic velocities in pure hydrocarbons and mixtures, J. Acoustic Society of America, 89, pp. 2725-2730.

Wood, A. (1930) Textbook of Sound, 1st Edition. 